\begin{tabular}{|c|c|c|c|}
\hline \multirow{3}{*}{$\begin{array}{r}\text { Case Reports in } \\
\text { Gastroenterology }\end{array}$} & \multirow{2}{*}{\multicolumn{2}{|c|}{ Case Rep Gastroenterol 2018;12:217-223 }} & \multirow[b]{3}{*}{$\begin{array}{l}\text { Karger } \\
\text { Open access }\end{array}$} \\
\hline & & & \\
\hline & $\begin{array}{l}\text { DOI: } 10.1159 / 000489296 \\
\text { Published online: May 31, } 2018\end{array}$ & $\begin{array}{l}\text { (c) } 2018 \text { The Author(s) } \\
\text { Published by S. Karger AG, Basel } \\
\text { www.karger.com/crg }\end{array}$ & \\
\hline & $\begin{array}{l}\text { This article is licensed under } \\
\text { International License (CC BY-N } \\
\text { Usage and distribution for comm }\end{array}$ & $\begin{array}{l}\text { mons Attribution-NonCommercial } 4.0 \\
\text { rger.com/Services/OpenAccessLicense). } \\
\text { quires written permission. }\end{array}$ & \\
\hline
\end{tabular}

\title{
Hypothermia-Related Acute Pancreatitis
}

\author{
Kyawzaw Lin ${ }^{a}$ Emmanuel Oforib Aung Naing Lin ${ }^{\mathrm{a}}$ Sithu Lin ${ }^{\mathrm{a}}$ \\ Thinzar Lin ${ }^{a} \quad$ Ameer Rasheed $^{c}$ Viswanath Vasudevan ${ }^{d} \quad$ Madhavi Reddy $^{\mathrm{e}}$ \\ aDepartment of Internal Medicine, The Brooklyn Hospital Center, Affiliate of the Mount \\ Sinai Hospital, Brooklyn, NY, USA; ${ }^{b} \mathrm{GI}$ Department, The Brooklyn Hospital Center, Affiliate \\ of the Mount Sinai Hospital, Brooklyn, NY, USA; 'MICU, The Brooklyn Hospital Center, \\ Affiliate of the Mount Sinai Hospital, Brooklyn, NY, USA; ${ }^{d}$ Critical Care Department, The \\ Brooklyn Hospital Center, Affiliate of the Mount Sinai Hospital, Brooklyn, NY, USA; \\ e $G$ I Department, The Brooklyn Hospital Center, Affiliate of the Mount Sinai Hospital, \\ Brooklyn, NY, USA
}

\section{Keywords}

Hypothermia · Acute pancreatitis · Hepatobiliary · Gastroenterology

\begin{abstract}
Acute pancreatitis (AP) is an inflammatory disease presenting from mild localized inflammation to severe infected necrotic pancreatic tissue. In the literature, there are a few cases of hypothermia-induced AP. However, the association between hypothermia and AP is still a myth. Generally, mortality from acute pancreatitis is nearly $3-6 \%$. Here, we present a 40 -year-old chronic alcoholic female who presented with acute pancreatitis induced by transient hypothermia. A 40-year-old chronic alcoholic female was hypothermic at $81^{\circ} \mathrm{F}$ on arrival which was improved to $91.7^{\circ} \mathrm{F}$ with warming blanket and then around $97^{\circ} \mathrm{F}$ in $8 \mathrm{~h}$. Laboratory tests including complete blood count, lipid panel, and comprehensive metabolic panels were within the normal limit. Serum alcohol level was 0.01 , amylase 498 , lipase 1,200 , ammonia 26 , serum carboxyhemoglobin level 2.4, and $\beta-H C G$ was negative. The entire sepsis workup was negative. During rewarming period, she had one episode of witnessed generalized tonic-clonic seizure. It was followed by transient hypotension. Fluid challenge was successful with $2 \mathrm{~L}$ of normal saline. Sonogram (abdomen) showed fatty liver and trace ascites. CAT scan (abdomen and pelvis) showed evidence of acute pancreatitis without necrosis, peripancreatic abscess, pancreatic
\end{abstract}


mass, or radiopaque gallstones. The patient was managed medically and later discharged from the hospital on the 4th day as she tolerated a normal low-fat diet. In our patient, transient hypothermia from chronic alcohol abuse and her social circumstances might predispose to microcirculatory disturbance resulting in acute pancreatitis. Early and aggressive fluid resuscitation prevents complications.

(C) 2018 The Author(s)

Published by S. Karger AG, Basel

\section{Introduction}

Acute pancreatitis (AP) is an inflammatory disease presenting from mild localized inflammation to severe infected necrotic pancreatic tissue. AP is the most common cause of gastrointestinal-related acute hospital admissions in the USA. Premature activation of trypsinogen to trypsin in pancreatic acinar cell is the triggering event for AP. AP is usually diagnosed by 2 of 3 criteria: (i) constant epigastric or upper abdominal pain that radiated to the back, flanks, or chest, (ii) serum amylase and/or lipase 3 times higher than the upper normal limit, and (iii) characteristic findings on computed tomography (CT). The mortality rate from AP is approximately 3-6\%. The common causes of AP are gallstones, alcohol, biliary sludge, medications such as 6-mercaptopurine, azathioprine, and dideoxyinosine, metabolic causes including hypercalcemia, hypertriglyceridemia, and hyperparathyroidism, and infections such as mumps. Serum triglyceride level should be considered as a cause in the absence of gallstones or alcohol consumption. Pancreatic tumor is another cause of AP in the elderly population (older than 40 years). However, genetic testing should be done in the young population if there is no obvious cause for AP and there is a family history of pancreatic pathology. Transabdominal sonography should be done in all patients with AP. Hemodynamic status together with hematocrits and blood urea nitrogen should be reassessed at regular intervals beginning at admission. Timely adequate fluid replacement is the mainstream of management. AP with acute cholangitis should be managed with ERCP within $24 \mathrm{~h}$ of admission. Antibiotics play a role only for extrapancreatic sepsis in the setting of bacteremia, urinary tract infection, biliary infection, pneumonia, or catheter-related infections. The patients usually respond to early and adequate fluid resuscitation and conservative medical management. Infected pancreatic necrosis should be borne in mind in patients with AP or extrapancreatic necrosis who suddenly deteriorate, do not respond to optimal management, or have increasing demand for vasopressors in intensive care settings, usually in 7-10 days of hospital admission. In the literature, there are a few cases of hypothermia-induced AP. However, the association between hypothermia and AP is still a myth. The two most common causes of AP are gallstones and alcohol. However, in our patient, AP was caused by transient hypothermia. Here, we present a 40-yearold chronic alcoholic female presenting with transient hypothermia-related AP.

\section{Case Report}

A 40-year-old chronic alcoholic female was brought by EMS to the emergency department after being found sleeping in a car on the street. The patient admitted drinking alcohol on a daily basis and her last drink was $1 \mathrm{~h}$ prior to arrival. She was hypothermic at $81^{\circ} \mathrm{F}$ on arrival which was improved to $91.7^{\circ} \mathrm{F}$ with a warming blanket and then around $97^{\circ} \mathrm{F}$ in $8 \mathrm{~h}$. Initial blood tests showed white blood cell count of 9.1 with mild left shift, hemoglobin 11, hematocrit 34 , platelets 458 , serum alcohol level 0.01 , creatinine phosphokinase 564 , blood urea nitrogen 16 , creatinine 0.4 , glucose 58 , aspartate transaminase 188 , alanine transaminase 69 , 
alkaline phosphatase 216, TSH 1.07, prolactin 44.9, amylase 498, lipase 1,200, ammonia 26, serum carboxyhemoglobin level 2.4, magnesium 1.3, cortisol 38, and $\beta$-HCG negative. During the rewarming period, she had one episode of witnessed generalized tonic-clonic seizure and was managed with intravenous lorazepam $2 \mathrm{mg}$ and a loading dose of levetiracetam 1,000 mg. It was followed by transient hypotension. Fluid challenge was successful after $2 \mathrm{~L}$ of normal saline. Vancomycin, cefepime, and metronidazole were given empirically for possible septic shock. Later, the full sepsis workup was negative and antibiotics were held off. A sonogram (abdomen) showed fatty liver and trace ascites (Fig. 1). A CAT scan (abdomen and pelvis) showed no radiopaque gallstones. Peripancreatic fluid was seen as well as fluid in the splenic flexure of the colon and inferior aspect of the spleen. The pancreas itself was enhanced symmetrically and there was no evidence of pancreatic necrosis, hemorrhage, peripancreatic abscess, or pancreatic mass (Fig. 2). She was managed medically and later discharged from hospital on the 4th day as she tolerated a low-fiber low-fat diet.

\section{Discussion}

AP was the most common gastrointestinal-related hospital admission diagnosis with a health care financial burden of USD 2.6 billion in the USA in 2009. The incidence is about $10-$ 100 per 100,000 population. The incidence of AP has been seen to be on the rise recently but the case fatality rate from AP has decreased over time. However, the general population mortality rate has reportedly remained unchanged. The overall mortality rate in AP is 3-6\% while in severe pancreatitis it is $30 \%$ [1].

Two common causes are gallstones (40-70\%) and alcohol (25-35\%). These are followed by biliary sludge, medications (such as 6-mercaptopurine, azathioprine, and dideoxyinosine), metabolic causes (including hypercalcemia, hypertriglyceridemia, and hyperparathyroidism), and infections such as mumps. Either primary or secondary hypertriglyceridemia can cause AP but only accounts for $1-4 \%$ of cases. In such cases, serum triglyceride needs to be more than $1,000 \mathrm{mg} / \mathrm{dL}[2]$.

Several case reports of AP from accidental hypothermia have been recorded in the literature. The clinical presentation varies from mild AP to severe hemorrhagic pancreatitis. AP is a common presentation in the adult population with accidental hypothermia. Serum lipase and amylase levels are high in 50-65\% of hypothermic patients while AP is found to be 20$30 \%$ at autopsy. However, the exact pathogenic mechanism of hypothermia-induced pancreatitis is poorly recognized [3].

Vascular endothelium instability, increased vascular permeability, vasomotor autoregulation disturbance, and activation of coagulation cascade can result in early complications of AP. Thus, fluid sequestration is maximal in $48 \mathrm{~h}$ after the onset of AP. It is associated with systemic inflammatory response syndrome and subsequent multi-organ failures in both moderately severe and severe AP [4].

There are 2 phases of AP: (i) early phase (less than a week) presents with systemic inflammatory response syndrome with/without single or multi-organ failure and (ii) late phase (more than a week) usually presents with local complications: peripancreatic fluid collections, pancreatic necrosis, pancreatic pseudocysts, and walled-off necrosis.

The diagnosis is made by the presence of 2 of 3 criteria: (i) constant epigastric or upper abdominal pain that radiates to back, flanks, or chest, (ii) serum amylase and/or lipase 3 times higher than the upper normal limit, and (iii) characteristic findings on CT. Contrast-enhanced CT and MRI must be considered for uncertain diagnosis or those unresponsive to optimal 
management in 48-72 $\mathrm{h}$ after hospital admission. MRI is another option for patients with contrast allergy and end-stage renal disease [5]. Transabdominal sonogram is a must in all cases of AP.

Serum lipase is preferred over amylase as it is more specific and remains longer in the serum. Serum amylase level can be normal in AP in the setting of alcohol-induced and hypertriglyceridemia-induced AP. Both serum amylase and lipase can falsely be high in nonpancreatic conditions such as acute appendicitis, cholecystitis, intestinal ischemia or obstruction, renal disease, and macro-amylasemia. Serum triglyceride level should be checked in the absence of alcohol abuse or history of gallstones.

D-Dimer and angiopoietin-2 can potentially be checked to foretell the severity of AP. Increased angiopoietin-2 level is positively correlated with the severity of AP, sepsis, multiorgan failure, mortality, bowel ischemia, and possibility of kidney injury [6]. von Willebrand factor concentration is also directly correlated with the severity of multi-organ failure, risk of acute lung injury, and sequential organ failure assessment score [7]. Osteoprotegerin level is higher in severe AP compared to mild AP [8].

According to the Atlanta revision criteria (2013), patients with moderately severe AP (local complications with/without transient organ failure $<48 \mathrm{~h}$ ) or severe AP (persistent organ failure $>48 \mathrm{~h}$ ), C-reactive protein $>10 \mathrm{mg} / \mathrm{dL}$, Ranson score $\geq 4$, Acute Physiology and Chronic Health Evaluation (APACHE) II score $\geq 9$, and Bedside Index of Severity in Acute Pancreatitis (BISAP) score $\geq 2$ should be admitted to an intensive care unit.

Early aggressive hydration, preferably with lactate ringer, should always be set up within the first 12-24 h unless there are underlying comorbidities such as heart failure and end-stage kidney disease. Hemodynamic status along with close monitoring of hematocrits and blood urea nitrogen should be reassessed at regular intervals within $6 \mathrm{~h}$ of admission and following $24-48 \mathrm{~h}[9,10]$.

Unfortunately, after so many randomized controlled trials, no effective medication has been reported for AP. Timely intravenous fluid replacement is the mainstream of management. For better electrolyte balance and optimal outcomes, more $\mathrm{pH}$-balanced lactate ringer is preferred over normal saline as low $\mathrm{pH}$ activates trypsinogen to trypsin in acinar cells - the triggering event for AP [11].

Antibiotics are useful for decreasing mortality and morbidity, and delaying or avoiding unnecessary surgical intervention. Antibiotics including quinolones, carbapenems and metronidazole are only indicated for extrapancreatic infections such as cholangitis, bacteremia, pneumonia urinary tract infection, and infected pancreatic necrosis. However, based on current guidelines, prophylactic antibiotics or probiotics have no role in patients with severe AP or patients with sterile necrosis $[12,13]$.

For infected pancreatic necrosis, management that should be considered is either initial CT-guided fine-needle aspiration for aspirated fluids for appropriate antibiotics or empirical antibiotics without fine-needle aspiration [14].

In mild AP, oral feeding can be started as early as possible when there is no abdominal pain and the patient can tolerate a liquid diet without nausea and vomiting. Prolonged placement of the pancreas at rest and prolonged bowel rest is correlated with a high chance of transmucosal bacterial translocation in the setting of intestinal mucosa atrophy. Multiple studies have emphasized that early oral feeding in AP shortens the hospital stay, reduces infectious complications, and decreases mortality and morbidity [15].

In moderately severe and severe AP, enteral feeding (either nasogastric or nasojejunal route) is preferred but parenteral feeding should be avoided. In patients with AP in the setting of gallstones, interval cholecystectomy must be done before the patient is discharged home. 
In conclusion, in the literature, there are a few cases of hypothermia-induced AP. However, the association between hypothermia and AP is still a myth. In our patient, transient hypothermia from chronic alcohol abuse and her social circumstances might predispose to microcirculatory disturbance resulting in AP. Early and aggressive fluid resuscitation prevents complications. We here report a case intended to remind all emergency department and intensive care unit physicians to keep a high suspicious index for AP in all hypothermic patients.

\section{Statement of Ethics}

Patient consent was obtained for this report.

\section{Disclosure Statement}

The authors declare no conflicts of interest.

\section{Author Contributions}

Dr. Kyawzaw Lin contributed to gathering information and writing the introduction, case report, and discussion. Dr. Aung Naing Lin, Dr. Sithu Lin, Dr. Emmanuel Ofori, and Dr. Ameer Rasheed contributed to editing the paper. Dr. Viswanath Vasudevan and Dr. Madhavi Reddy provided optimal patient care and management of the case. All authors contributed equally and approved the submitted manuscript.

\section{References}

1 Fagenholz PJ et al. Direct medical costs of acute pancreatitis hospitalizations in the United States. Pancreas. 2007 Nov;35(4):302-7.

2 Fortson MR, Freedman SN, Webster PD 3rd. Clinical assessment of hyperlipidemic pancreatitis. Am J Gastroenterol. 1995 Dec;90(12):2134-9.

3 Maclean D, Murison J, Griffiths PD. Acute pancreatitis and diabetic ketoacidosis in accidental hypothermia and hypothermic myxoedema. BMJ. 1973 Dec;4(5895):757-61.

4 de-Madaria E, Banks PA, Moya-Hoyo N, Wu BU, Rey-Riveiro M, Acevedo-Piedra NG et al. Early factors associated with fluid sequestration and outcomes of patients with acute pancreatitis. Clin Gastroenterol Hepatol. 2014 Jun;12(6):997-1002.

5 Štimac D, Miletić D, Radić M, Krznarić I, Mazur-Grbac M, Perković D et al. The role of nonenhanced magnetic resonance imaging in the early assessment of acute pancreatitis. Am J Gastroenterol. 2007 May;102(5):9971004.

6 Adler DG, Verma D, Hilden K, Chadha R, Thomas K. Dye-free wire-guided cannulation of the biliary tree during ERCP is associated with high success and low complication rates: outcomes in a single operator experience of 822 cases. J Clin Gastroenterol. 2010 Mar;44(3):e57-62.

7 Siemiatkowski A, Wereszczynska-Siemiatkowska U, Mroczko B, Galar M, Maziewski T. Circulating endothelial mediators in human pancreatitis-associated lung injury. Eur J Gastroenterol Hepatol. 2015 Jun;27(6):728-34.

8 Dumnicka P, Zylka A, Kusnierz-Cabala B, Gurda-Duda A, Kuzniewski M, Drozdz R et al. Osteoprotegerin, trail and osteoprotegerin/trail ratio in patients at early phase of acute pancreatitis. Folia Med Cracov. 2014;54(2):17-26.

9 Tenner S. Initial management of acute pancreatitis: critical issues during the first 72 hours. Am J Gastroenterol. 2004 Dec;99(12):2489-94. 
Lin et al.: Hypothermia-Related Acute Pancreatitis

10 Brown A, Orav J, Banks PA. Hemoconcentration is an early marker for organ failure and necrotizing pancreatitis. Pancreas. 2000 May;20(4):367-72.

11 Wu BU, Hwang JQ, Gardner TH, Repas K, Delee R, Yu S et al. Lactated Ringer's solution reduces systemic inflammation compared with saline in patients with acute pancreatitis. Clin Gastroenterol Hepatol. 2011 Aug;9(8):710-717.e1.

12 Jiang K et al. Present and future of prophylactic antibiotics for severe acute pancreatitis. World J Gastroenterol. 2012 Jan;18(3):279-84.

13 Sun S, Yang K, He X, Tian J, Ma B, Jiang L. Probiotics in patients with severe acute pancreatitis: a metaanalysis. Langenbecks Arch Surg. 2009 Jan;394(1):171-7.

14 Freeman ML, Werner J, van Santvoort HC, Baron TH, Besselink MG, Windsor JA et al.; International Multidisciplinary Panel of Speakers and Moderators. Interventions for necrotizing pancreatitis: summary of a multidisciplinary consensus conference. Pancreas. 2012 Nov;41(8):1176-94.

15 Louie BE et al. 2004 MacLean-Mueller Prize enteral or parenteral nutrition for severe pancreatitis: a randomized controlled trial and health technology assessment. Can J Surg 48.42005 Aug;48(4):298-306.

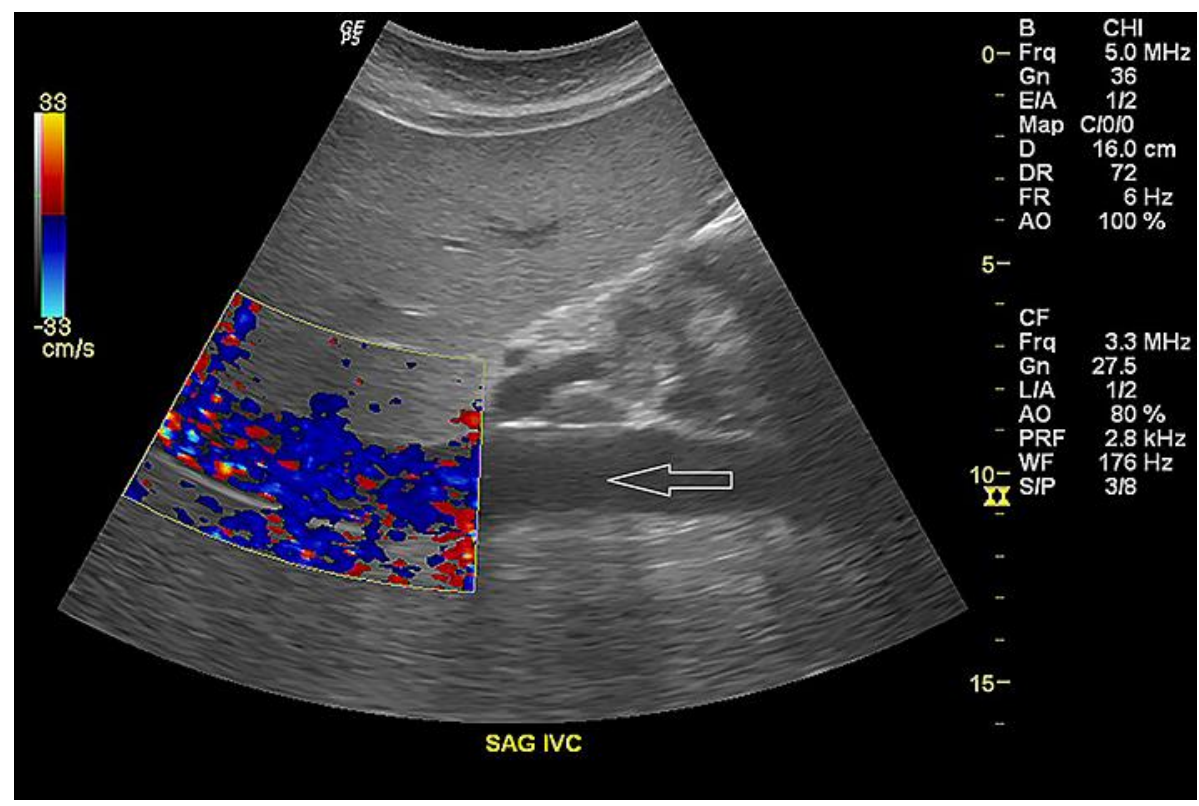

Fig. 1. Sonogram (right upper quadrant) showing hepatomegaly with echogenic appearance of hepatic parenchyma compatible with fatty infiltration or hepatocellular disease. Trace ascites can be seen (white arrow). 


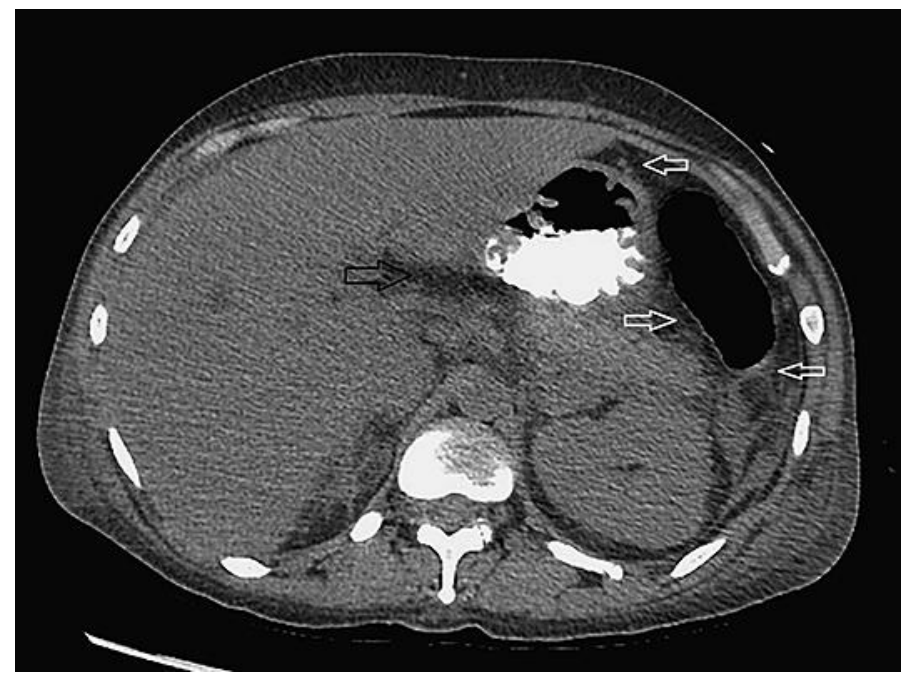

Fig. 2. CT scan (abdomen) showing thickening of the wall of the stomach that could be related to the patient's pancreatitis. There is peripancreatic fluid (black arrow) and fluid adjacent to the splenic flexure, the inferior aspect of the spleen, and left pericolic gutter (white arrows). The pancreas itself is symmetrically enhanced with no evidence of pancreatic necrosis, peripancreatic abscess/hemorrhage, or pancreatic mass. 